\title{
AN ELLIPTIC PROBLEM WITH CRITICAL EXPONENT AND POSITIVE HARDY POTENTIAL
}

\author{
SHAOWEI CHEN AND SHUJIE LI
}

Received 9 September 2003

We give the existence result and the vanishing order of the solution in 0 for the following equation: $-\triangle u(x)+\left(\mu /|x|^{2}\right) u(x)=\lambda u(x)+u^{2^{*}-1}(x)$, where $x \in B_{1}, \mu>0$, and the potential $\mu /|x|^{2}-\lambda$ is positive in $B_{1}$.

\section{Introduction}

In this paper, we consider the following problem:

$$
\begin{aligned}
-\triangle u(x)+\frac{\mu}{|x|^{2}} u(x) & =\lambda u(x)+u^{2^{*}-1}(x), \quad x \in B_{1}, \\
u(x) & \geq 0, \quad x \in B_{1}, \\
u(x) & =0, \quad x \in \partial B_{1},
\end{aligned}
$$

where $B_{1}=\left\{x \in \mathbb{R}^{N}|| x \mid<1\right\}$ is the unit ball in $\mathbb{R}^{N}(N \geq 3), \lambda, \mu>0,2^{*}:=2 N /(N-2)$. When $\mu<0$, this problem has been considered by many authors recently (cf. $[5,6,7$, $8]$ ). But when $\mu>0$, this problem has not been considered as far as we know. In fact, the existence of nontrivial solution for (1.1) when $\mu>0$ is an open problem which was imposed in [7]. In this paper, we get the following results.

Theorem 1.1. If $N=3$ and $3 / 4<\lambda \leq \mu$ or if $N \geq 4$ and $0<\lambda \leq \mu$, then for (1.1) there exists a nontrivial radially symmetric solution.

Remark 1.2. Condition $0<\lambda \leq \mu$ shows that the potential $\mu /|x|^{2}-\lambda$ is positive in $B_{1}$. Thus the Brézis-Nirenberg method (cf. [1]) cannot be used.

THEOREM 1.3. If $\mu>0$ and $u \in H_{0}^{1}\left(B_{1}\right)$ is a solution of (1.1), then there are $C_{1}, C_{2}>0$ and $\delta>0$ such that $C_{2}|x|^{\alpha} \geq u(x) \geq C_{1}|x|^{\alpha}$, for $x \in B_{\delta}$, where $\alpha=(1 / 2)\left(\sqrt{(N-2)^{2}+4 \mu^{2}}-\right.$ $(N-2))>0$.

Remark 1.4. One can easily deduce that if $u \in H_{0}^{1}\left(B_{1}\right)$ is a solution of (1.1), then $u \in$ $C^{2}\left(B_{1} \backslash\{\theta\}\right)$ and $u>0$ in $B_{1} \backslash\{\theta\}$. Theorem 1.3 shows that $u(\theta)=0$. It is greatly different from the case of $\mu \leq 0$ (see [6]). 


\section{Proof of Theorem 1.1}

LEMMA 2.1. Every radially symmetric nonnegative solution $u$ of the equation

$$
-\triangle u+\frac{\mu}{|x|^{2}} u(x)=u^{2^{*}-1}(x), \quad u \in \mathscr{D}^{1,2}\left(\mathbb{R}^{N}\right)
$$

can be represented by $u(x)=\rho^{(N-2) / 2} U(\rho x)$ for some positive number $\rho$, where

$$
U(x)=\frac{C_{0}|x|^{\tau-(N-2) / 2}}{\left(1+|x|^{4 \tau /(N-2)}\right)^{(N-2) / 2}},
$$

$\tau=\sqrt{((N-2) / 2)^{2}+\mu}$, and $C_{0}$ is a constant.

Proof. Let $t=-\ln |x|, \theta=x /|x|$, and $v(t, \theta):=e^{-((N-2) / 2) t} u\left(e^{-t} \theta\right)$. Then by [3], we know that $v$ satisfies the equation

$$
-v_{t t}-\triangle_{\theta} v+\tau^{2} v=v^{2^{*}-1} \quad \text { in } \mathbb{R} \times \mathbb{S}^{N-1}
$$

Since $u$ is radially symmetric, $v$ depends only on $t$ and satisfies $-v_{t t}+\tau^{2} v=v^{2^{*}-1}, v>0$ in $\mathbb{R}$. By [3], we know that the only positive solutions of the equation are translation of

$$
v(t)=\left(\frac{\tau^{2} 2^{*}}{2}\right)^{1 /\left(2^{*}-1\right)}\left(\cosh \left(\frac{2^{*}-2}{2} \tau t\right)\right)^{-2 /\left(2^{*}-2\right)}
$$

Thus, every radially symmetric nonnegative solution $u$ of (2.1) can be represented by $u(x)=\rho^{(N-2) / 2} U(\rho x)$ for some positive number $\rho$.

Define $\mathscr{D}_{r}^{1,2}\left(\mathbb{R}^{N}\right):=\left\{u \in \mathscr{D}^{1,2}\left(\mathbb{R}^{N}\right) \mid u\right.$ is radially symmetric $\}$ and $H_{0, r}^{1}\left(B_{1}\right):=\{u \in$ $H_{0}^{1}\left(B_{1}\right) \mid u$ is radially symmetric $\}$. Let

$$
S_{\mu}:=\inf _{u \in \mathscr{D}_{r}^{1,2}\left(\mathbb{R}^{N}\right), u \neq 0} \frac{\int_{\mathbb{R}^{N}}|\nabla u|^{2}+\mu \int_{\mathbb{R}^{N}}\left(u^{2} /|x|^{2}\right)}{\left(\int_{\mathbb{R}^{N}}|u|^{2 *}\right)^{2 / 2^{*}}} .
$$

It follows from Lemma 2.1 that $S_{\mu}=\left(\int_{\mathbb{R}^{N}}|\nabla U|^{2}+\mu \int_{\mathbb{R}^{N}}\left(U^{2} /|x|^{2}\right)\right) /\left(\int_{\mathbb{R}^{N}} U^{2^{*}}\right)^{2 / 2^{*}}$. Let $\Sigma=$ $\left\{u \in H_{0, r}^{1}\left(B_{1}\right) \mid\|u\|_{2^{*}}=1\right\}$. For $u \in \Sigma$, define

$$
S_{\lambda, \mu}(u)=\int_{B_{1}}|\nabla u|^{2}+\mu \int_{B_{1}} \frac{u^{2}}{|x|^{2}}-\lambda \int_{B_{1}} u^{2}
$$

LEMMA 2.2. If $N=3$ and $3 / 4<\lambda \leq \mu$ or if $N \geq 4$ and $0<\lambda \leq \mu$, then $S_{\lambda, \mu}:=\inf _{u \in \Sigma} S_{\lambda, \mu}(u)<$ $S_{\mu}$. 
Proof. Let $\eta \in C_{0}^{\infty}\left(\mathbb{R}^{N}\right)$ be a cut function which satisfies $0 \leq \eta(x) \leq 1,|\nabla \eta| \leq 2$ in $\mathbb{R}^{N}$, $\eta(x) \equiv 1$ in $B_{1 / 2}$, and $\eta(x) \equiv 0$ in $\mathbb{R}^{N} \backslash B_{1}$. Let $U_{\rho}(x):=\rho^{(N-2) / 2} U(\rho x)$ and $u_{\rho}(x)=\eta(x) U_{\rho}(x)$. By (2.2), we know that when $|x|$ is big enough, there are constants $C_{1}, C_{2}>0$ such that

$$
|U(x)| \leq \frac{C_{1}}{|x|^{\tau+N / 2-1}}, \quad|\nabla U(x)| \leq \frac{C_{2}}{|x|^{\tau+N / 2}}
$$

since

$$
\begin{aligned}
\int_{B_{1}}\left|\nabla u_{\rho}\right|^{2}= & \int_{B_{1}} \eta^{2}\left|\nabla u_{\rho}\right|^{2}+\int_{B_{1}} u_{\rho}^{2}|\nabla \eta|^{2}+2 \int_{B_{1}} u_{\rho} \cdot \eta \cdot \nabla u_{\rho} \cdot \nabla \eta \\
& \leq \int_{B_{1}}\left|\nabla u_{\rho}\right|^{2}+4 \int_{B_{1} \backslash B_{1 / 2}} u_{\rho}^{2}+4\left(\int_{B_{1} \backslash B_{1 / 2}} u_{\rho}^{2}\right)^{1 / 2}\left(\int_{B_{1} \backslash B_{1 / 2}}\left|\nabla u_{\rho}\right|^{2}\right)^{1 / 2} \\
& =\int_{\mathbb{R}^{N}}|\nabla U|^{2}+\int_{\mathbb{R}^{N} \backslash B_{\rho}}|\nabla U|^{2}+\frac{4}{\rho^{2}} \int_{B_{\rho} \backslash B_{\rho / 2}} U^{2} \\
& +\frac{4}{\rho}\left(\int_{B_{\rho} \backslash B_{\rho / 2}} U^{2}\right)^{1 / 2}\left(\int_{B_{\rho} \backslash B_{\rho / 2}}|\nabla U|^{2}\right)^{1 / 2} .
\end{aligned}
$$

By (2.7), when $N=3$ and $3 / 4<\lambda \leq \mu$ or when $N \geq 4$ and $0<\lambda \leq \mu$, for $\rho$ big enough,

$$
\begin{gathered}
\int_{B_{\rho} \backslash B_{\rho / 2}} U^{2} \leq \int_{B_{\rho} \backslash B_{\rho / 2}} \frac{C_{1}}{|x|^{2 \tau+N-2}} d x=\frac{C_{3}}{\rho^{2 \tau-2}}, \\
\int_{\mathbb{R}^{N} \backslash B_{\rho}}|\nabla U|^{2} \leq \int_{\mathbb{R}^{N} \backslash B_{\rho}} \frac{C_{2}}{|x|^{2 \tau+N}} d x=\int_{\rho}^{+\infty} \frac{C_{2}}{r^{2 \tau+1}} d r=\frac{C_{4}}{\rho^{2 \tau}}, \\
\int_{B_{1}}\left|\nabla u_{\rho}\right|^{2} \leq \int_{\mathbb{R}^{N}}|\nabla U|^{2}+\frac{C_{5}}{\rho^{2 \tau}}, \\
\int_{B_{1}} \frac{u_{\rho}^{2}}{|x|^{2}} \leq \int_{\mathbb{R}^{N}} \frac{U^{2}}{|x|^{2}}+\frac{C_{6}}{\rho^{2 \tau}}, \quad \int_{B_{1}}\left|u_{\rho}\right|^{2^{*}} \geq \int_{\mathbb{R}^{N}} U^{2^{*}}-\frac{C_{7}}{\rho^{2 * \tau}}, \\
\int_{B_{1}} u_{\rho}^{2} \geq \frac{C_{8}}{\rho^{2}} .
\end{gathered}
$$

When $N=3$ and $3 / 4<\lambda \leq \mu$ or when $N \geq 4$ and $0<\lambda \leq \mu$, we have $2 \tau>2$. Thus by $(2.10)$ and (2.11), we get

$$
S_{\lambda, \mu} \frac{u_{\rho}}{\left\|u_{\rho}\right\|_{2^{*}}} \leq S_{\mu}-\frac{C_{9}}{\rho^{2}}+o\left(\frac{1}{\rho^{2}}\right), \quad \text { as } \rho \longrightarrow \infty .
$$

It proves the lemma.

Proof of Theorem 1.1. By Lemma 2.2 and [10, Theorem 8.8], we deduce that $S_{\lambda, \mu}$ can be achieved by some $0 \leq u \in H_{0, r}^{1}\left(B_{1}\right)$, then $S_{\lambda, \mu}^{-1 /\left(2^{*}-2\right)} u$ is a nontrivial radially symmetric solution of (1.1). 


\section{Proof of Theorem 1.3}

Let $E$ be the space which is the completion of $C_{0}^{\infty}\left(B_{1}\right)$ under the norm $\|u\|_{E}=$ $\left(\int_{B_{1}}|x|^{2 \alpha}|\nabla u|^{2} d x\right)^{1 / 2}$.

Lemma 3.1 (see [2]). For all $u \in C_{0}^{\infty}\left(\mathbb{R}^{N}\right)(N \geq 3)$,

$$
\left(\int_{\mathbb{R}^{N}}|x|^{-b p}|u|^{p} d x\right)^{2 / p} \leq C_{a, b} \int_{\mathbb{R}^{N}}|x|^{-2 a}|\nabla u|^{2} d x
$$

where $-\infty<a<(N-2) / 2, a \leq b \leq a+1$, and $p=2 N /(N-2+2(b-a))$.

Choosing $a=-\alpha, p=2$ and $2^{*}$, respectively, in (3.1), we get the following lemma.

Lemma 3.2. There is a constant $C>0$ such that, for any $u \in C_{0}^{\infty}\left(\mathbb{R}^{N}\right)$,

$$
\begin{aligned}
\left(\int_{\mathbb{R}^{N}}|x|^{2^{*} \alpha}|u|^{2^{*}} d x\right)^{2 / 2^{*}} & \leq C \int_{\mathbb{R}^{N}}|x|^{2 \alpha}|\nabla u|^{2} d x, \\
\int_{\mathbb{R}^{N}}|x|^{2 \alpha-2}|u|^{2} d x & \leq C \int_{\mathbb{R}^{N}}|x|^{2 \alpha}|\nabla u|^{2} d x .
\end{aligned}
$$

Proof of Theorem 1.3. If $v \in H_{0}^{1}\left(B_{1}\right)$ is a solution of (1.1), then by the standard regularity theory, one can easily deduce that $v \in C^{2}\left(B_{1} \backslash\{\theta\}\right)$. Let $u(x)=|x|^{-\alpha} v(x)$ (this kind of transform has been used in [9]). Direct calculation shows that, for any $x \in B_{1} \backslash\{\theta\}$,

$$
-\operatorname{div}\left(|x|^{2 \alpha} \nabla u\right)=|x|^{2^{*} \alpha} u^{2^{*}-1}+\lambda|x|^{2 \alpha} u
$$

Since $v \in E$, then by Lemma 3.1 we know that $v$ is a weak solution of (3.3), that is, for any $\zeta \in C_{0}^{\infty}\left(B_{1}\right)$

$$
\int_{B_{1}}|x|^{2 \alpha} \nabla u \nabla \zeta=\int_{B_{1}}|x|^{2^{*} \alpha} u^{2^{*}-1} \zeta+\int_{B_{1}}|x|^{2 \alpha} u \zeta
$$

For $t>2, k>0$, define

$$
h(r)= \begin{cases}r^{t / 2}, & 0 \leq r \leq k, \\ \frac{t}{2} k^{t / 2-1} r+\left(1-\frac{t}{2}\right) k^{t / 2}, & r \geq k,\end{cases}
$$

and $\phi(r)=\int_{0}^{r}\left|h^{\prime}(s)\right|^{2} d s$. It is easy to verify that there exists a constant $C>0$ independent of $k$ such that

$$
\begin{gathered}
|r \phi(r)| \leq \frac{t^{2}}{4(t-1)}|h(r)|^{2}, \\
\left|\phi(r)-h(r) h^{\prime}(r)\right| \leq C_{t}\left|h(r) h^{\prime}(r)\right|,
\end{gathered}
$$

where $C_{t}=(t-2) / 2(t-1)<1$. 
Let $0<r_{2}<r_{1}<1$ and $\eta \in C_{0}^{\infty}\left(B\left(\theta, r_{1}\right)\right)$ satisfying $0 \leq \eta \leq 1, \eta \equiv 1$ in $B\left(\theta, r_{2}\right), \eta \equiv 0$ in $\mathbb{R}^{N} \backslash B\left(\theta, r_{1}\right)$, and $|\nabla \eta| \leq 2 /\left(r_{1}-r_{2}\right)$. Notice that $\eta^{2} \phi(u) \in E$, then

$$
\begin{aligned}
\int_{B_{1}}|x|^{2 \alpha} \nabla u \nabla\left(\eta^{2} \phi(u)\right) & =\int_{B_{1}}|x|^{2 \alpha} \eta^{2}\left(h^{\prime}(u)\right)^{2}|\nabla u|^{2}+2 \int_{B_{1}}|x|^{2 \alpha} \eta \phi(u) \nabla u \nabla \eta \\
& =\int_{B_{1}}|x|^{2 \alpha} \eta^{2}|\nabla(h(u))|^{2}+2 \int_{B_{1}}|x|^{2 \alpha} \eta \phi(u) \nabla u \nabla \eta .
\end{aligned}
$$

Since $|\nabla(\eta h(u))|^{2}=\eta^{2}|\nabla(h(u))|^{2}+h^{2}(u)|\nabla \eta|^{2}+2 \eta h(u) \nabla(h(u)) \nabla \eta$, by (3.7), we have

$$
\begin{aligned}
\int_{B_{1}}|x|^{2 \alpha} \nabla u \nabla\left(\eta^{2} \phi(u)\right)= & \int_{B_{1}}|x|^{2 \alpha}|\nabla(\eta h(u))|^{2}-\int_{B_{1}}|x|^{2 \alpha} h^{2}(u)|\nabla \eta|^{2} \\
& -2 \int_{B_{1}}|x|^{2 \alpha} \eta h(u) h^{\prime}(u) \nabla u \nabla \eta+2 \int_{B_{1}}|x|^{2 \alpha} \eta \phi(u) \nabla u \nabla \eta \\
\geq & \int_{B_{1}}|x|^{2 \alpha}|\nabla(\eta h(u))|^{2}-\int_{B_{1}}|x|^{2 \alpha} h^{2}(u)|\nabla \eta|^{2} \\
& -2 \int_{B_{1}}|x|^{2 \alpha} \eta\left|\phi(u)-h(u) h^{\prime}(u)\right||\nabla u \nabla \eta| \\
\geq & \int_{B_{1}}|x|^{2 \alpha}|\nabla(\eta h(u))|^{2}-\int_{B_{1}}|x|^{2 \alpha} h^{2}(u)|\nabla \eta|^{2} \\
& -2 C_{t} \int_{B_{1}}|x|^{2 \alpha}|\eta h(u) \nabla(h(u)) \nabla \eta| .
\end{aligned}
$$

Since

$$
\begin{aligned}
\int_{B_{1}}|x|^{2 \alpha}|\eta h(u) \nabla(h(u)) \nabla \eta| & =\int_{B_{1}}|x|^{2 \alpha}|(\nabla(\eta h(u))-h(u) \nabla \eta) \nabla \eta||h(u)| \\
\leq & \int_{B_{1}}|x|^{2 \alpha}|h(u) \nabla(\eta h(u)) \nabla \eta|+\int_{B_{1}}|x|^{2 \alpha}|h(u)|^{2}|\nabla \eta|^{2} \\
\leq & \frac{1}{2} \int_{B_{1}}|x|^{2 \alpha} h^{2}(u)|\nabla \eta|^{2}+\frac{1}{2} \int_{B_{1}}|x|^{2 \alpha}|\nabla(\eta h(u))|^{2} \\
& +\int_{B_{1}}|x|^{2 \alpha}|h(u)|^{2}|\nabla \eta|^{2},
\end{aligned}
$$

and by (3.9), we deduce that

$$
\begin{aligned}
\int_{B_{1}}|x|^{2 \alpha} \nabla u \nabla\left(\eta^{2} \phi(u)\right) & \\
\geq & \int_{B_{1}}|x|^{2 \alpha}|\nabla(\eta h(u))|^{2}-\int_{B_{1}}|x|^{2 \alpha} h^{2}(u)|\nabla \eta|^{2} \\
& \quad-2 C_{t}\left(\frac{1}{2} \int_{B_{1}}|x|^{2 \alpha} h^{2}(u)|\nabla \eta|^{2}+\frac{1}{2} \int_{B_{1}}|x|^{2 \alpha}|\nabla(\eta h(u))|^{2}+\int_{B_{1}}|x|^{2 \alpha}|h(u)|^{2}|\nabla \eta|^{2}\right)
\end{aligned}
$$




$$
\begin{aligned}
& =\frac{t}{2(t-1)} \int_{B_{1}}|x|^{2 \alpha}|\nabla(\eta h(u))|^{2}-\left(1+3 C_{t}\right) \int_{B_{1}}|x|^{2 \alpha} h^{2}(u)|\nabla \eta|^{2} \\
& \geq \frac{C t}{2(t-1)}\left(\int_{B_{1}}|x|^{2^{*} \alpha}|\eta h(u)|^{2^{*}}\right)^{2 / 2^{*}}-\left(1+3 C_{t}\right) \int_{B_{1}}|x|^{2 \alpha} h^{2}(u)|\nabla \eta|^{2} .
\end{aligned}
$$

By (3.6), we have

$$
\begin{aligned}
\int_{B_{1}}|x|^{2^{*} \alpha} u^{2^{*}-1} \eta^{2} \phi(u)+\int_{B_{1}}|x|^{2 \alpha} u \eta^{2} \phi(u) \\
\leq \frac{t^{2}}{4(t-1)} \int_{B_{1}}|x|^{2^{*} \alpha}|u|^{2^{*}-2}|\eta h(u)|^{2}+\frac{t^{2}}{4(t-1)} \int_{B_{1}}|x|^{2 \alpha}|\eta h(u)|^{2} \\
\leq \frac{t^{2}}{4(t-1)}\left(\int_{\eta \neq 0}|x|^{2^{*} \alpha}|u|^{2^{*}}\right)^{\left(2^{*}-2\right) / 2^{*}}\left(\int_{B_{1}}|\eta h(u)|^{2^{*}}\right)^{2 / 2^{*}} \\
\quad+\frac{t^{2}}{4(t-1)} \int_{B_{1}}|x|^{2 \alpha}|\eta h(u)|^{2} .
\end{aligned}
$$

Notice that $u$ is a solution of (3.3), by (3.11) and (3.12) we have

$$
\begin{aligned}
\left(\int_{B_{1}}|x|^{2^{*} \alpha}|\eta h(u)|^{2^{*}}\right)^{2 / 2^{*}} & \\
\leq & \frac{t}{2 C}\left(\int_{\eta \neq 0}|x|^{2^{*} \alpha}|u|^{2^{*}}\right)^{\left(2^{*}-2\right) / 2^{*}}\left(\int_{B_{1}}|x|^{2^{*} \alpha}|\eta h(u)|^{2^{*}}\right)^{2 / 2^{*}} \\
& +\frac{2\left(1+3 C_{t}\right)(t-1)}{C t} \int_{B_{1}}|x|^{2 \alpha} h^{2}(u)|\nabla \eta|^{2}+\frac{t}{2 C} \int_{B_{1}}|x|^{2 \alpha}|\eta h(u)|^{2} .
\end{aligned}
$$

Choose $r_{1}$ small enough such that $(t / 2 C)\left(\int_{\eta \neq 0}|x|^{2^{*} \alpha}|u|^{2^{*}}\right)^{\left(2^{*}-2\right) / 2^{*}}<1 / 2$. Notice that $2(1+$ $\left.3 C_{t}\right)(t-1) / t<8$ (since $0<C_{t}<1$ and $\left.t>2\right)$ and $|\nabla \eta|<2 /\left(r_{1}-r_{2}\right)$, from (3.13) we have

$$
\left(\int_{B\left(\theta, r_{2}\right)}|x|^{2^{*} \alpha}|h(u)|^{2^{*}}\right)^{2 / 2^{*}} \leq\left(\frac{64}{C\left(r_{1}-r_{2}\right)^{2}}+\frac{t}{C}\right) \int_{B\left(\theta, r_{1}\right)}|x|^{2 \alpha} h^{2}(u) .
$$

Choosing $2(N-2 \alpha) /(N-2+2 \alpha)>t_{0}>2$ and letting $k \rightarrow \infty$ in (3.14), we get

$$
\left(\int_{B\left(\theta, r_{2}\right)}|x|^{2^{*} \alpha}|u|^{2^{*} t_{0} / 2}\right)^{2 / 2^{*}} \leq\left(\frac{64}{C\left(r_{1}-r_{2}\right)^{2}}+\frac{t_{0}}{C}\right) \int_{B\left(\theta, r_{1}\right)}|x|^{2 \alpha}|u|^{t_{0}}
$$

By Lemma 3.1, we know that $\left(\int_{B_{1}}|x|^{2 \alpha}|u|^{t_{0}}\right)^{2 / t_{0}} \leq \int_{B_{1}}|x|^{2 \alpha}|\nabla u|^{2}<\infty$. Combining (3.15), we get that

$$
\int_{B_{1}}|x|^{2^{*} \alpha}|u|^{2^{*} t_{0} / 2}<\infty
$$


Since

$$
\begin{aligned}
& \int_{B_{1}}|x|^{2 \alpha} \nabla u \nabla(\phi(u))=\int_{B_{1}}|x|^{2 \alpha}|\nabla(h(u))|^{2} \geq\left(\int_{B_{1}}|x|^{2^{*} \alpha}|h(u)|^{2^{*}}\right)^{2 / 2^{*}}, \\
& \int_{B_{1}}|x|^{2^{*} \alpha} u^{2^{*}-1} \phi(u)+\int_{B_{1}}|x|^{2 \alpha} u \phi(u) \\
& \leq \frac{t^{2}}{4(t-1)} \int_{B_{1}}|x|^{2^{*} \alpha}|u|^{2^{*}-2}|h(u)|^{2}+\frac{t^{2}}{4(t-1)} \int_{B_{1}}|x|^{2 \alpha}|h(u)|^{2} \\
& \leq \frac{t^{2}}{4(t-1)}\left(\int_{B_{1}}|x|^{2^{*} \alpha}|u|^{2^{*} t_{0} / 2}\right)^{2\left(2^{*}-2\right) / 2^{*} t_{0}}\left(\int_{B_{1}}|x|^{2^{*} \alpha}|h(u)|^{q}\right)^{2 / q} \\
&+\frac{t^{2}}{4(t-1)} \int_{B_{1}}|x|^{2 \alpha}|h(u)|^{2} \\
& \leq \frac{t^{2}}{4(t-1)}\left(\int_{B_{1}}|x|^{2^{*} \alpha}|u|^{2^{*} t_{0} / 2}\right)^{2\left(2^{*}-2\right) / 2^{*} t_{0}}\left(\int_{B_{1}}|x|^{2^{*} \alpha}|h(u)|^{q}\right)^{2 / q} \\
&+\frac{t^{2}}{4(t-1)}\left(\int_{B_{1}}|x|^{\left(2 \alpha-2^{*} \alpha / q\right) q^{\prime}}\right)^{1 / q^{\prime}}\left(\int_{B_{1}}|x|^{2^{*} \alpha}|h(u)|^{q}\right)^{2 / q},
\end{aligned}
$$

where $q=2 \cdot 2^{*} t_{0} /\left(\left(t_{0}-2\right) 2^{*}+4\right)$ and $2 / q+1 / q^{\prime}=1$, we can deduce that if $\epsilon>0$ small enough and $t_{0} \in(2,2+\epsilon)$, then $\left(2 \alpha-2^{*} \alpha / q\right) q^{\prime}>-2$. Thus $\left(\int_{B_{1}}|x|^{\left(2 \alpha-2^{*} \alpha / q\right) q^{\prime}}\right)^{1 / q^{\prime}}<\infty$. Let $C^{\prime}=\left(\int_{B_{1}}|x|^{2^{*} \alpha}|u|^{2^{*} t_{0} / 2}\right)^{2\left(2^{*}-2\right) / 2^{*} t_{0}}+\left(\int_{B_{1}}|x|^{\left(2 \alpha-2^{*} \alpha / q\right) q^{\prime}}\right)^{1 / q^{\prime}}$, then by (3.17), we have

$$
\left(\int_{B_{1}}|x|^{2^{*} \alpha}|h(u)|^{2^{*}}\right)^{2 / 2^{*}} \leq \frac{C^{\prime} t^{2}}{4(t-1)}\left(\int_{B_{1}}|x|^{2^{*} \alpha}|h(u)|^{q}\right)^{2 / q} .
$$

Letting $k \rightarrow \infty$, we get

$$
|u|_{2^{*} t / 2,2^{*} \alpha} \leq\left(\frac{C^{\prime} t^{2}}{4(t-1)}\right)^{1 / t}|u|_{q t / 2,2^{*} \alpha}
$$

where $|u|_{l, 2^{*} \alpha}:=\left(\int_{B_{1}}|x|^{2^{*} \alpha}|u|^{l}\right)^{1 / l}$.

Choose $t_{1}=(2 * / q)^{n}, n=1,2, \ldots$. Then by (3.19) we have

$$
|u|_{2 * t_{n} / 2,2 * \alpha} \leq \prod_{i=1}^{n}\left(\frac{C^{\prime} t_{i}^{2}}{4\left(t_{i}-1\right)}\right)^{1 / t_{i}}|u|_{2 * / 2,2 * \alpha} .
$$

Letting $n \rightarrow \infty$, we deduce that $u \in L^{\infty}\left(B_{1}\right)$. Thus there is $C_{2}>0$ such that $v(x) \leq C_{2}|x|^{\alpha}$.

Since $\operatorname{div}\left(|x|^{2 \alpha} \nabla u\right) \leq 0$, by [4, Lemma 4.2], we have $u(x) \geq C^{\prime \prime}>0$ for $x \in B_{\delta}$. So, there is $C_{1}>0$ such that $u(x) \geq C_{1}|x|^{\alpha}$ for $x \in B_{\delta}$. 


\section{References}

[1] H. Brézis and L. Nirenberg, Positive solutions of nonlinear elliptic equations involving critical Sobolev exponents, Comm. Pure Appl. Math. 36 (1983), no. 4, 437-477.

[2] L. A. Caffarelli, R. Kohn, and L. Nirenberg, First order interpolation inequalities with weights, Compositio Math. 53 (1984), no. 3, 259-275.

[3] F. Catrina and Z.-Q. Wang, On the Caffarelli-Kohn-Nirenberg inequalities: sharp constants, existence (and nonexistence), and symmetry of extremal functions, Comm. Pure Appl. Math. 54 (2001), no. 2, 229-258.

[4] K. S. Chou and C. W. Chu, On the best constant for a weighted Sobolev-Hardy inequality, J. London Math. Soc. (2) 48 (1993), no. 1, 137-151.

[5] K. S. Chou and D. Geng, On the critical dimension of a semilinear degenerate elliptic equation involving critical Sobolev-Hardy exponent, Nonlinear Anal. 26 (1996), no. 12, 1965-1984.

[6] A. Ferrero and F. Gazzola, Existence of solutions for singular critical growth semilinear elliptic equations, J. Differential Equations 177 (2001), no. 2, 494-522.

[7] E. Jannelli, The role played by space dimension in elliptic critical problems, J. Differential Equations 156 (1999), no. 2, 407-426.

[8] D. Ruiz and M. Willem, Elliptic problems with critical exponents and Hardy potentials, J. Differential Equations 190 (2003), no. 2, 524-538.

[9] D. Smets and A. Tesei, On a class of singular elliptic problems with first order terms, Adv. Differential Equations 8 (2003), no. 3, 257-278.

[10] M. Willem, Minimax Theorems, Progress in Nonlinear Differential Equations and Their Applications, vol. 24, Birkhäuser Boston, Massachusetts, 1996.

Shaowei Chen: Institute of Mathematics, Academy of Mathematics and System Sciences, Chinese Academy of Sciences, Beijing 100080, China

E-mail address: chensw@mail.amss.ac.cn

Shujie Li: Institute of Mathematics, Academy of Mathematics and System Sciences, Chinese Academy of Sciences, Beijing 100080, China

E-mail address: lisj@math.ac.cn 


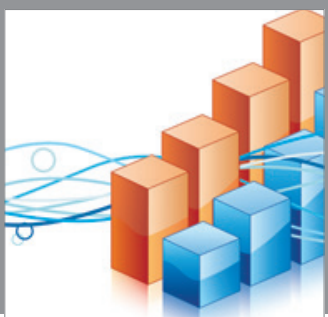

Advances in

Operations Research

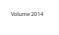

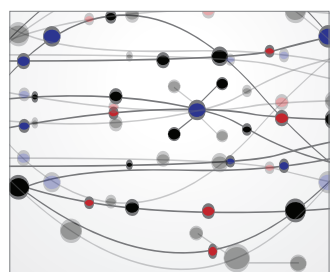

\section{The Scientific} World Journal
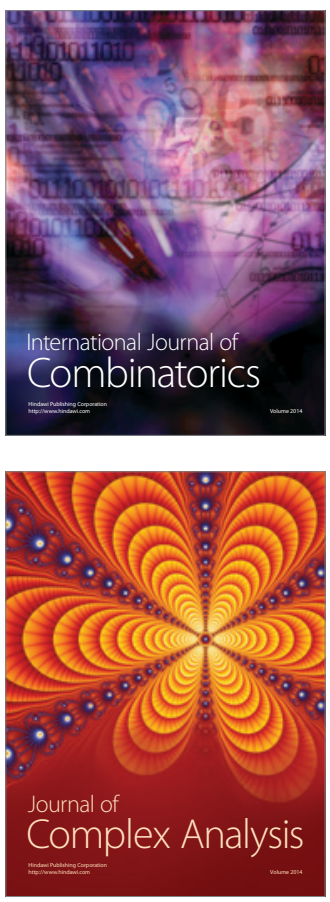

International Journal of

Mathematics and

Mathematical

Sciences
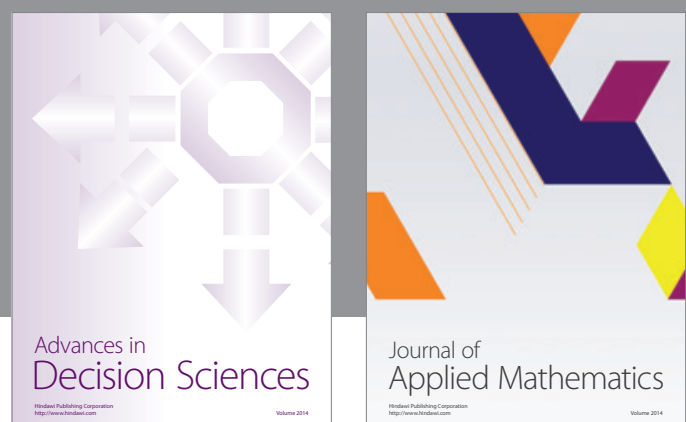

Journal of

Applied Mathematics
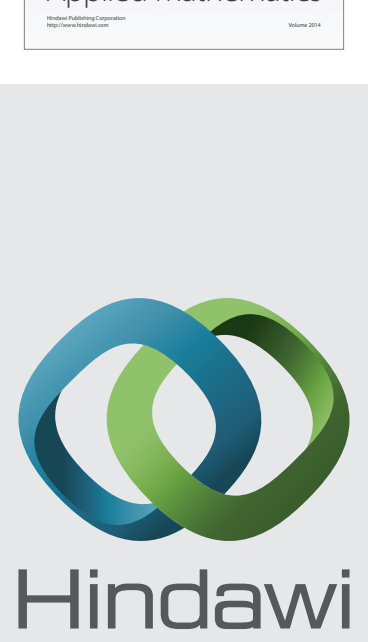

Submit your manuscripts at http://www.hindawi.com
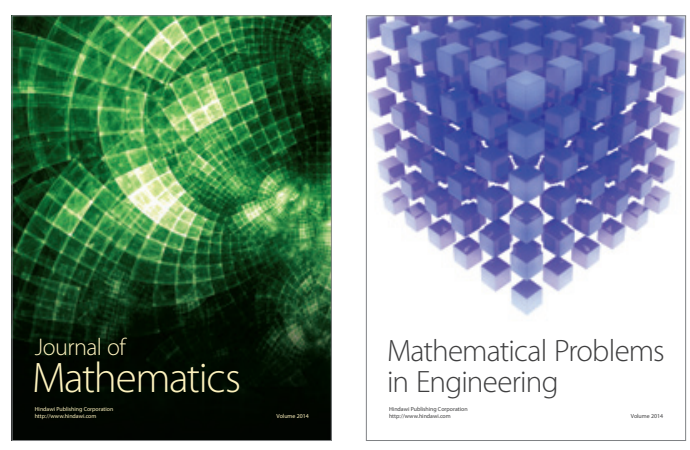

Mathematical Problems in Engineering
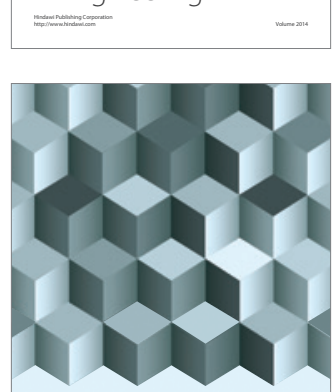

Journal of

Function Spaces
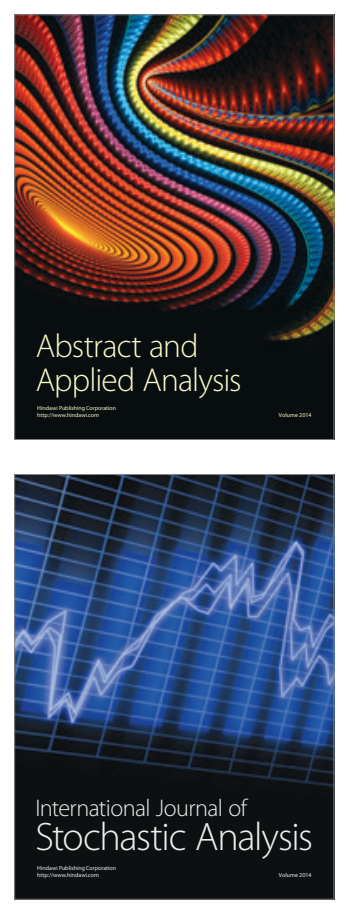

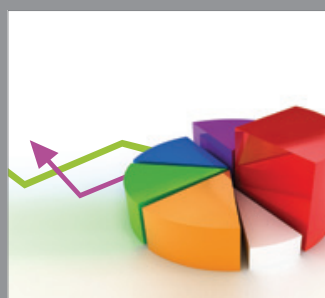

ournal of

Probability and Statistics

Promensencen
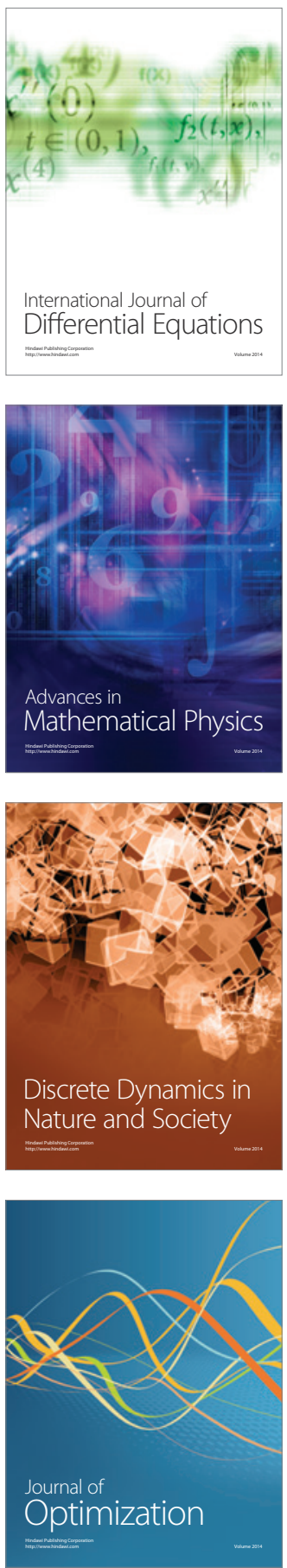